\title{
Consumo de alcohol y violencia doméstica contra las mujeres: un estudio con estudiantes universitarias de México
}

\author{
José Manuel Herrera Paredes ${ }^{1}$ \\ Carla Aparecida Arena Ventura ${ }^{2}$
}

La violencia doméstica contra la mujer y el consumo de alcohol son considerados problemas de salud pública. Esta investigación descriptiva presentó como objetivo general determinar la ocurrencia de violencia doméstica contra mujeres estudiantes de una Facultad de Enfermería y Obstetricia de la Ciudad de Celaya, en Guanajuato, México, y su relación con el consumo de alcohol por las parejas de estas mujeres. La muestra fue compuesta por 73 alumnas, seleccionadas aleatoriamente. Para efectos de recolección de datos, se utilizó el cuestionario estructurado y validado denominado CASIQUE-QUEJ TUN. Los datos fueron analizados con el paquete estadístico SPPS. En lo que se refiere a la violencia física, 91,9\% manifestaron no haber sido maltratadas por sus parejas. En lo que se refiere al consumo de alcohol, $57,5 \%$ de las entrevistadas y $67,1 \%$ de sus parejas lo consumen en reuniones sociales. Los resultados apuntaron también que $41,1 \%$ de las participantes no conocen sus derechos con relación a la violencia doméstica. De esta forma, son necesarias acciones de concientización de las mujeres sobre sus derechos, y las posibilidades de buscar ayuda legal en casos de violencia.

Descriptores: Violencia; Derechos de la Mujer; Alcoholismo.

\footnotetext{
${ }^{1}$ Enfermero, Facultad de Enfermería y Obstetricia de Celaya, Universidad de Guanajuato, México. E-mail: jmanuelherrer@ hotmail.com y jmanuelherrer@yahoo.com.

2 Profesor Doctor, Escola de Enfermagem de Ribeirão Preto, Universidade de São Paulo, Centro Colaborador de la OMS para el Desarrollo de la Investigación en Enfermería, Brasil. E-mail: caaventu@eerp.usp.br.
}

Correspondencia:

Carla Aparecida Arena Ventura

Universidade de São Paulo. Escola de Enfermagem de Ribeirão Preto

Av. Bandeirantes, 3900

Bairro Monte Alegre

CEP: 14040-902 Ribeirão Preto, SP, Brasi

E-mail: caaventu@eerp.usp.br 


\title{
Consumo de álcool e violência doméstica contra a mulher: estudo com estudantes universitárias mexicanas
}

A violência doméstica contra a mulher e o consumo de álcool são considerados problemas de saúde pública. Esta pesquisa descritiva apresentou como objetivo geral determinar a ocorrência de violência doméstica contra mulheres, alunas da Faculdade de Enfermagem e Obstetrícia da cidade de Celaya, México, e sua relação com o consumo de álcool por seus parceiros íntimos. A amostra foi composta por 73 alunas, selecionadas aleatoriamente. Para a coleta de dados, foi utilizado o questionário estruturado e validado, denominado CASIQUE-QUEJ TUN. Os dados foram analisados através do programa SPSS. Quanto à violência física, 91,9\% manifestaram não ter sido maltratadas por seus parceiros. Com relação ao consumo de álcool, $57,5 \%$ das entrevistadas e $67,1 \%$ de seus parceiros consomem álcool, especialmente em reuniões sociais. Os resultados apontaram também que $41,1 \%$ não conhecem seus direitos em relação à violência doméstica. Dessa forma, são necessárias ações de conscientização das mulheres sobre seus direitos e possibilidades de ajuda legal em casos de violência.

Descritores: Violência; Direitos da Mulher; Alcoolismo.

\section{Alcohol Consumption and Domestic Violence Against Women: a Study with University Students from Mexico}

\begin{abstract}
Domestic violence against women and alcohol consumption are considered public health problems. This descriptive study aimed at determining the occurrence of domestic violence against women enrolled in the Nursing and Midwifery Program at the University of Celaya, Mexico and its relation with alcohol consumption by their partners. A sample of 73 students was randomly selected. Data were collected using the CASIQUE-QUEJ TUN questionnaire and analyzed using SPSS. With respect to physical violence, results showed that $91.9 \%$ were not maltreated by their partners. Regarding alcohol consumption, $57.5 \%$ of the women interviewed and $67.1 \%$ of their partners drank alcohol, especially at social events. Results also showed that $41.1 \%$ of the subjects did not know about their rights in cases of domestic violence. Thus, there is a need for actions which increase women's awareness of their rights and the possibilities of legal support in cases of violence.
\end{abstract}

Descriptors: Violence; Women's Rights; Alcoholism.

\section{Introducción}

En México, las drogas no son un fenómeno nuevo y aunque el índice de consumo no sea tan elevado como en otros países, desde 1976 hasta la presente fecha, dichos índices han ido aumentando tanto en frecuencia como en extensión dentro de la comunidad estudiantil, en el ámbito del bachillerato y universitario. Entre todas las drogas, el uso de alcohol constituye un grave problema para la salud pública, con consecuencias personales, familiares y sociales.

En la actualidad, el alcohol es la droga preferida por los jóvenes, la que es considerada sumamente peligrosa, pues su consumo está asociado a una mayor probabilidad de tener problemas en la escuela, estar sexualmente activos, estar implicados en accidentes de tráfico y ser víctimas de crímenes violentos. En este contexto se ha observado que uno de los efectos del consumo de alcohol es la violencia(1).

La conducta violenta, entendida como el uso de la fuerza para la resolución de conflictos personales, surge cuando existe un desequilibrio de poder, permanente o momentáneo, en el cual se establece una relación de abuso. Por medio de ese tipo de conducta se busca 
someter, doblegar o subordinar al otro miembro de la relación, ocasionándole daño físico, psíquico, económico, social y/o sexual(2).

La relación entre consumo de alcohol y conductas violentas constituye un problema social con repercusiones legales. La violencia y el maltrato familiar se han reconocido como un problema socialmente vinculado al consumo de alcohol, principalmente consumido por el hombre, quien ejecuta algún tipo de violencia en la mujer. Específicamente sobre la violencia contra la mujer, los datos mundiales revelan que ocurre en todos los países; esta es aplicada en gran parte de la población femenina, tanto adulta como infantil, en una magnitud de carácter endémico, siendo manifestada de varias formas y en los más diversos espacios de la sociedad, independientemente de la clase social, edad, raza, tipo de cultura o grado de desarrollo económico del país. Siendo así, hay muchos estudios que afirman ser el espacio doméstico el lugar en que más ocurren agresiones contra la mujer, con resultados perjudiciales para su salud mental y física(3).

Considerando la importancia y complejidad del fenómeno de la violencia contra la mujer, esta investigación presentó como objetivo general determinar la ocurrencia de violencia doméstica contra mujeres estudiantes de una Facultad de Enfermería y Obstetricia de la Ciudad de Celaya, en Guanajuato, México, y su relación con el consumo de alcohol por las parejas de estas mujeres.

\section{Drogas, Alcohol y Violencia en México: efectos para la salud de las mujeres}

Según la Organización Mundial de la Salud (OMS), droga es toda sustancia que poco tiempo después de ser incorporada al organismo produce modificación en sus funciones $^{(4)}$ y la drogadicción es la imperiosa necesidad que una persona tiene de consumir drogas.

En México las drogas son una problemática que va aumentando día a día, involucrando a la población menor de edad. Se dice que de cada 100 mexicanos, de 10 a 15 tienen problemas con el consumo de drogas ilegales o alcohol(5).

El alcoholismo es una entidad clínica definida como adicción al alcohol de las bebidas embriagantes. Se manifiesta a través de dependencia, tolerancia y síndrome de abstinencia. Como trastorno mental, aparece por primera vez reconocido en la clasificación de enfermedades de la Asociación Psiquiátrica Americana en 1980, posteriormente es introducido en la Clasificación Internacional de Enfermedades, en el apartado de Trastornos Mentales y del Comportamiento debido al uso de sustancias psicoactivas. Se expresa por la necesidad compulsiva de consumir alcohol y la dificultad para abandonarlo constituyendo una forma de dependencia, como la que se evidencia en otras adicciones(4).

El alcoholismo figura entre los diez principales problemas de salud pública en el mundo, siendo el cuarto que produce más incapacidades. De acuerdo a los datos de la OMS, el alcohol es la sustancia psicoactiva más utilizada. Con relación a la dependencia de alcohol, la prevalencia entre hombres es de tres a cuatro veces mayor que en las mujeres ${ }^{(6)}$. Muchos estudios asocian el consumo de alcohol a un aumento de la violencia doméstica, especialmente la sufrida por mujeres ${ }^{(3-4,7)}$ y perpetrada por sus parejas ${ }^{(8)}$.

La violencia es una acción ejercida por una o varias personas en donde se somete de manera intencional al maltrato, presión, sufrimiento, manipulación u otra acción que atente contra la integridad tanto física como psicológica y moral de cualquier persona o grupo de personas. Por lo tanto, "la violencia es la presión síquica o abuso de la fuerza ejercida contra una persona con el propósito de obtener fines contra la voluntad de la víctima"(1). La conducta violenta es aquella que provoca daño físico o emocional en quien la experimenta y se genera principalmente por las diferencias entre los géneros. En la década de 1980, la violencia contra la mujer fue inicialmente reconocida por la OMS como un problema de salud pública, por su magnitud y por la gravedad de las secuelas orgánicas y emocionales producidas $^{(9)}$. En este sentido, algunos estudios llaman la atención para los impactos de la violencia en salud física y mental de las mujeres(10-12).

Existen diversas formas de violencia, la física que abarca una escala de conductas que van desde un empujón o un pellizco hasta lesiones graves que llevan a la muerte; la violencia psicológica, que incluye conductas como criticar permanentemente el cuerpo o las ideas, hacer comparaciones con otras personas, cuestionar todo lo que hace y cómo lo hace; la violencia verbal que consiste en descalificar a la mujer y restarle autoridad frente a los hijos, criticar a su familia o a personas que ella estima; la violencia sexual, en que se obliga a la mujer a la realización de conductas sexuales no deseadas; y, la violencia económica, en que se excluye a la mujer de la toma de decisiones financieras, controlando sus gastos ${ }^{(8)}$. Este estudio fue centrado en las violencias física, psicológica, verbal y sexual.

Dentro de los factores de riesgo para la violencia son apuntados: experiencia de violencia en la infancia, condiciones de exclusión social, pobreza, vivir en guerra prolongada $\mathrm{y}$, tener acceso a armas, drogas y alcohol(13). 
Siendo así, el alcoholismo registra un gran porcentaje de situaciones en que las mujeres son agredidas por sus compañeros conyugales, que están bajo el efecto del alcohol. De esta forma, se verifica una mayor vulnerabilidad femenina frente a la violencia familiar y también que la presencia del alcohol en el hogar se relaciona de manera importante con la agresión familiar, principalmente contra las mujeres, siendo una variable de riesgo(14). Las consecuencias de la violencia contra la mujer pueden no ser mortales y adoptar la forma de lesiones físicas a discapacidad crónica o problemas de salud mental. También pueden ser mortales; ya sea por homicidio o por muerte como resultado de lesiones permanentes. Además de las consecuencias físicas, se observan muchos problemas psicológicos, como la baja autoestima y el aumento de la vulnerabilidad a las enfermedades. Mujeres que sufren violencia doméstica están cinco veces más predispuestas a presentaren problemas psicológicos que las mujeres que no viven esa situación. Alteraciones como nerviosismo, ansiedad y cambios de sueño son informados por mujeres que sufren violencia. Las mujeres en situación de violencia también están más vulnerables a abortos, violación sexual y enfermedades sexualmente transmisibles ${ }^{(15)}$.

En el ámbito de las legislaciones nacionales basadas en el derecho humano a la integridad física, las conductas violentas son consideradas ilegales y generan sanciones a los infractores. En México, la sanción que se impone al agresor puede ir de seis meses a cuatro años de prisión, independientemente de las sanciones que correspondan a las lesiones inferidas por cualquier otro delito. A las personas que sufren violencia dentro de la ley federal, se les sugiere que acudan inmediatamente a la Agencia del Ministerio Público más cercana y exijan que se inicie una averiguación previa por violencia familiar. Así, en México hay una ley específica que protege a las mujeres que sufren violencia doméstica, puniendo sus agresores.

\section{Procedimientos Metodológicos}

Este estudio fue de tipo descriptivo, transversal, cuantitativo y exploratorio realizado entre Noviembre de 2006 y Julio de 2007. Tomaron parte las 294 alumnas de la carrera de enfermería, la muestra aleatoria simple fue compuesta por 73 alumnas, seleccionadas aleatoriamente de acuerdo con los criterios de elegibilidad. Los criterios de inclusión fueron: ser del sexo femenino, desear participar en el estudio y pertenecer como alumnas del programa de Licenciatura en Enfermería y Obstetricia del sistema escolarizado de una Facultad de Enfermería y Obstetricia de Celaya, en Guanajuato, México.

Para efectos de la recolección de los datos, se utilizó el cuestionario estructurado y validado (con una confiabilidad de alpha de Cronbach de 0,8), denominado CASIQUE-QUEJ TUN ${ }^{(16)}$, estructurado en dos partes: 1) Datos generales del sujeto del estudio, conteniendo 8 preguntas y, 2) Presencia de Violencia compuesto por 38 ítems de opción múltiple, organizado en una escala tipo Likert (siempre, a veces, rara vez, nunca).

Al instrumento se le realizaron ajustes en cuanto a datos generales relacionados con las siguientes preguntas: ¿ocupación?, ¿escolaridad? y ¿número de hijos muertos?, es decir; fue eliminadas las preguntas sobre ocupación y número de hijos muertos, en cuanto a la de escolaridad sólo se le realizó una adaptación para clasificar por el semestre en que se encuentra el sujeto de estudio, debido a que la recolección de datos se realizó en una institución educativa de licenciatura. Se agregó una encuesta que contenía 8 preguntas relacionadas con el consumo de alcohol, y una parte donde se incluye la repercusión legal.

Con la autorización del Comité de Ética de la Institución Universitaria donde se llevó a cabo la recolección de los datos, fueron identificadas las alumnas de la Licenciatura en Enfermería y Obstetricia que reunieron los criterios de inclusión. Se entrevistaron a las alumnas donde se informó sobre los objetivos del estudio, con la finalidad de solicitar y obtener su consentimiento libre e informado para la participación en el estudio. Se otorgó y se autoaplicó el instrumento de recolección de datos, dejando claramente la privacidad y el compromiso de confidencialidad, así como abierta la posibilidad de retirarse del estudio en cualquier momento en que ellos lo desearan, sin ninguna consecuencia para ellos. En el final del proceso, se revisaron los cuestionarios autoaplicados para verificar se habían contestado por completo el instrumento.

Los datos de los cuestionarios fueron analizados con el paquete estadístico SPPS para Windows, versión $11.0 \mathrm{y}$, tratados estadísticamente en forma descriptiva obteniendo frecuencias y porcentajes.

\section{Resultados}

De la muestra de 73 sujetos, el $100 \%$ corresponde al sexo femenino. El rango de edad está entre 18 y 29 años, con un promedio de 20,93 $\pm 2,03$ años. Con relación a las características sociodemográficas, 91,8\% eran solteras, $6,8 \%$ casadas y $1,4 \%$ informaron unión libre; $6,8 \%$ estaban en el 10 semestre de Licenciatura en Enfermería y Obstetricia, $24,7 \%$ en el $2^{\circ}$ semestre, $27,4 \%$ en el 40 semestre, $24,7 \%$ en el $6^{\circ}$ semestre y $16,4 \%$ en el $8^{\circ}$ semestre; $87,7 \%$ eran católicas, $91,8 \%$ no tenían hijos, 6,1\% tenían 1 hijo y 1,4\% 2 hijos; 
$43,8 \%$ convivían con la pareja por lo menos durante un año, 31,5\% entre 1 y 3 años, 20,5\% entre 4 y 6 años y $4,1 \%$ entre 7 y 10 años. Siendo así, la mayoría de las alumnas eran solteras, cursando del $2^{\circ}$ al $6^{\circ}$ semestre de Licenciatura en Enfermería y Obstetricia, católicas, sin hijos, y con un tiempo de convivencia con sus parejas de menos de tres años.

En cuanto a la presencia de violencia física, el $91,8 \%$ refirió nunca haber sido maltratada por su pareja, sin embargo se encontró un 2,7\%, 1,4\% y $4,1 \%$ que refieren siempre, a veces y rara vez respectivamente experimentar conductas agresivas por parte de su pareja. Once mujeres $(15,1 \%)$ expresaron de forma escrita sentirse controladas constantemente por su pareja y haber sufrido un jalón de cabellos, el $2,7 \%$ refirió a veces y de igual manera el 2,7\% refirió siempre. El $100 \%$ de la población negó que su pareja la haya lanzado contra la pared, el suelo o los muebles y el haber usado la fuerza física con la finalidad de tener relaciones sexuales con ella. La Tabla 1 presenta las preguntas relacionadas a violencia física y la frecuencia de respuestas por categorías.

Tabla 1 - Violencia Física sufrida por las alumnas por parte de su pareja. Celaya, Guanajuato, México; 2007

\begin{tabular}{|c|c|c|c|c|c|c|c|c|}
\hline \multirow{3}{*}{ Pregunta } & \multicolumn{8}{|c|}{ * Categoría } \\
\hline & \multicolumn{2}{|c|}{$\mathbf{S}$} & \multicolumn{2}{|c|}{ AV } & \multicolumn{2}{|c|}{ RV } & \multicolumn{2}{|c|}{$\mathbf{N}$} \\
\hline & $\mathbf{f}$ & $\%$ & $\mathbf{f}$ & $\%$ & f & $\%$ & f & $\%$ \\
\hline ¿Usted es maltratada o golpeada por su pareja? & 2 & 2,7 & 1 & 1,4 & 3 & 4,1 & 67 & 91,8 \\
\hline ¿Siente que su pareja constantemente la está controlando? & 2 & 2,7 & 2 & 2,7 & 11 & 15,1 & 58 & 79,5 \\
\hline ¿Su pareja le ha jalado de los cabellos? & 2 & 2,7 & 2 & 2,7 & 11 & 15,1 & 58 & 79,5 \\
\hline ¿Ha usado la fuerza física para tener relaciones sexuales con usted? & - & - & - & - & - & - & 73 & 100 \\
\hline ¿Su pareja la ha lanzado contra la pared, el suelo o muebles? & - & - & - & - & - & - & 73 & 100 \\
\hline
\end{tabular}

*(S - siempre, AV - a veces, RV - Rara vez, N - nunca)

Sobre la violencia verbal, el 2,7\% de las mujeres entrevistadas refirieron recibir humillaciones verbales por parte de su pareja, el 9,6\% son acusadas de infidelidad o de tener amantes. El 1,4\% manifestó haber sido amenazada de hacerle daño o a sus hijos y $11 \%$ nunca decide lo que puede hacer o no hacer, $4,1 \%$ a veces y $2,7 \%$ rara vez. Entre los resultados fue observado que $6,8 \%$ de las mujeres refieren que después de un episodio violento, su pareja se muestra cariñoso y atento, prometiendo que nunca más volverá a golpearla, insultarla y que "todo cambiará". La violencia verbal de la muestra refirió que siempre(24,7\%) su pareja la criticaba y humillaba en público o en privado sobre su apariencia, su forma de ser y el modo en de realizar los quehaceres domésticos, $6,8 \%$ a veces y $2,7 \%$ rara vez. Cuanto a la libertad de relacionarse con otras personas, 9,6\% informó que rara vez ha perdido contacto con amigas, familiares, compañeras/os de trabajo para evitar que su pareja se enoje, $5,5 \%$ a veces y $1,4 \%$ siempre. La Tabla 2 sintetiza los datos relacionados a la violencia verbal.

Tabla 2 - Violencia Verbal sufrida por las alumnas por parte de sus parejas. Celaya, Guanajuato, México; 2007

\begin{tabular}{|c|c|c|c|c|c|c|c|c|}
\hline \multirow{3}{*}{ Pregunta } & \multicolumn{8}{|c|}{ * Categoría } \\
\hline & \multicolumn{2}{|c|}{$\mathbf{S}$} & \multicolumn{2}{|c|}{ AV } & \multicolumn{2}{|c|}{ RV } & \multicolumn{2}{|c|}{$\mathbf{N}$} \\
\hline & f & $\%$ & f & $\%$ & $\mathbf{f}$ & $\%$ & f & $\%$ \\
\hline ¿Su pareja la humilla verbalmente? & & & 1 & 1,4 & 2 & 2,7 & 70 & 95,9 \\
\hline ¿La acusa de infidelidad o de tener amantes? & 1 & 1,4 & 2 & 2,7 & 7 & 9,6 & 63 & 86,3 \\
\hline $\begin{array}{l}\text { ¿Su pareja la ha amenazado alguna vez en hacerle daño a usted (o a sus hijos o algún } \\
\text { familiar de usted)? }\end{array}$ & & & 1 & 1,4 & 1 & 1,4 & 71 & 97,3 \\
\hline ¿Usted decide lo que puede hacer o no hacer? & 60 & 82,2 & 3 & 4,1 & 2 & 2,7 & 8 & 11,0 \\
\hline $\begin{array}{l}\text { Después de un episodio violento, él se muestra cariñoso y atento, le regala cosas y le } \\
\text { promete que nunca más volverá a golpearla o insultarla y que "todo cambiará" }\end{array}$ & 1 & 1,4 & 2 & 2,7 & 5 & 6,8 & 65 & 89,0 \\
\hline ¿Controla estrictamente sus ingresos o el dinero que le entrega, originando discusiones? & 1 & 1,4 & 1 & 1,4 & 2 & 2,7 & 69 & 94,5 \\
\hline $\begin{array}{l}\text { ¿La critica y humilla, en público o en privado, sobre su apariencia, su forma de ser y el } \\
\text { modo en que realiza sus quehaceres domésticos? }\end{array}$ & 18 & 24,7 & 5 & 6,8 & 2 & 2,7 & 48 & 65,8 \\
\hline $\begin{array}{l}\text { ¿Su pareja actúa cariñosamente y evita ser violento con usted frente a las demás } \\
\text { personas? }\end{array}$ & & & & & 1 & 1,4 & 72 & 98,6 \\
\hline $\begin{array}{l}\text { ¿Ha perdido contacto con amigas, familiares, compañeras/os de trabajo para evitar que } \\
\text { su pareja se enoje? }\end{array}$ & 1 & 1,4 & 4 & 5,5 & 7 & 9,6 & 61 & 83,6 \\
\hline
\end{tabular}

*(S - siempre, AV - a veces, RV - Rara vez, N - nunca) 
En lo que dice respecto a la violencia sexual, $2,7 \%$ refirió que a veces su pareja la ha maltratado o maltrata durante las relaciones sexuales y $83,6 \%$ creen que nunca ceden a los requerimientos sexuales de su pareja por temor. De estas mujeres, el $2,7 \%$ y el $1,4 \%$ manifestaron ser obligadas a tener relaciones sexuales.
Todas las alumnas entrevistadas manifestaron nunca haber sido chantajeadas con dinero para acceder a los deseos sexuales de las parejas y 1,4\% manifestó que su pareja le ha pedido que realice cosas que no le agradaban durante el acto sexual. La Tabla 3 presenta los datos sobre la violencia sexual.

Tabla 3 - Violencia Sexual sufrida por las alumnas por parte de sus parejas. Celaya, Guanajuato, México; 2007

\begin{tabular}{|c|c|c|c|c|c|c|c|c|}
\hline \multirow{3}{*}{ Pregunta } & \multicolumn{8}{|c|}{ * Categoría } \\
\hline & \multicolumn{2}{|c|}{$\mathbf{S}$} & \multicolumn{2}{|c|}{ AV } & \multicolumn{2}{|c|}{ RV } & \multicolumn{2}{|c|}{$\mathbf{N}$} \\
\hline & f & $\%$ & $\mathbf{f}$ & $\%$ & $\mathbf{f}$ & $\%$ & f & $\%$ \\
\hline ¿Su pareja la ha maltratado o maltrata durante las relaciones sexuales? & & & 2 & 2,7 & & & 71 & 97,3 \\
\hline $\begin{array}{l}\text { ¿Usted cree que cede a los requerimientos sexuales de su pareja por } \\
\text { temor? }\end{array}$ & 1 & 1,4 & 4 & 5,5 & 7 & 9,6 & 61 & 83,6 \\
\hline $\begin{array}{l}\text { ¿Su pareja la ha chantajeado con no darle dinero si no accede a sus } \\
\text { deseos sexuales? }\end{array}$ & & & & & & & 73 & 100 \\
\hline $\begin{array}{l}\text { ¿Su pareja le ha pedido que realice cosas que no le agradan durante el } \\
\text { acto sexual? }\end{array}$ & & & & & 1 & 1,4 & 72 & 98,6 \\
\hline $\begin{array}{l}\text { ¿Su pareja la obliga o ha obligado a tener relaciones sexuales sin su } \\
\text { consentimiento? }\end{array}$ & & & 2 & 2,7 & 1 & 1,4 & 70 & 95,9 \\
\hline
\end{tabular}

*(S - siempre, AV - a veces, RV - Rara vez, N - nunca)

Como marcas físicas de la violencia vivida, 2,7\% de las participantes refirieron que su pareja le han provocado algún tipo de fractura en alguna parte del cuerpo. El $4,1 \%$ y el $1,4 \%$ manifestaron que a veces y rara vez respectivamente su pareja la ha dado una bofetada. Con relación a la violencia psicológica, el 17,8\% dijo que rara vez su pareja consigue algo que desea, a través de hacerse la víctima, el 4,1\% refirió que a veces y el 2,7 que siempre lo hace. El 2,7\% refirió que siempre su que pareja quiere que cambie de comportamiento, la presiona con el silencio, con la indiferencia o la priva de dinero.

En relación al consumo de alcohol, 57,5\% de las mujeres entrevistadas consumen alcohol. El principal motivo por el consumo es por asistir a reuniones sociales $(49,3 \%)$ y el $4,1 \%$ refirió consumir para sentirse alegre.

En lo que se refiere al consumo de alcohol por parte de la pareja de las mujeres, se aprecia en los resultados que $67,1 \%$ de las parejas consumen alcohol. El principal motivo para el consumo es asistir a reuniones sociales $(53,4 \%)$, el $4,1 \%$ refirió consumir por ser tradición familiar el consumo alcohol y, el 1,4\% manifestó consumirlo cuando siente ansiedad y cuando está triste.

Sobre la búsqueda de ayuda externa, 100\% de las mujeres entrevistadas no han buscado ayuda de la policía, tampoco de un abogado o más información sobre cómo protegerse de su pareja, ya que no consideran haber sufrido violencia grave o "no ser necesario pedir ayuda a la policía". El 58,9\% refieren conocer los derechos con relación a la violencia doméstica.

\section{Discusión}

A pesar de que las organizaciones internacionales como la OMS y el Banco Mundial así como los profesionales de la salud (Asociación Médica Americana) divulgan elevadas prevalencias de violencia doméstica contra la mujer causada por parejas(17-18), en este estudio fue verificado un bajo índice de violencia física informado por las alumnas de Enfermería y Obstetricia de la Universidad de Guanajuato, lo que puede estar asociado al poco tiempo de convivencia con sus parejas y también al hecho de que solamente $19,2 \%$ de las mujeres entrevistadas vivían en la misma casa con sus parejas, una vez que la convivencia más cercana facilita la ocurrencia de conflictos que pueden generar conductas violentas. Las agresiones causadas por las parejas son así reconocidas como la forma más común de violencia contra la mujer; la que presenta el mayor riesgo de ser agredida física y sexualmente por quién convive íntimamente con ella más de que cualquier otra persona ${ }^{(19)}$. El bajo índice de violencia física también puede ser resultado de la llamada "invisibilidad" de los eventos violentos por las propias mujeres, que naturalizan y relativizan las violencias que sufren o no lo perciben como tal(20).

Se debe resaltar que el hecho de que la mayoría de las mujeres entrevistadas eran solteras también influyó en el resultado, una vez que la violencia representa uno 
de los mecanismos de dominación del hombre sobre la mujer, legitimado por instituciones como la familia y el casamiento. En esta dinámica, al destinar para la mujer un rol sumiso y pasivo, la sociedad crea un espacio para la dominación masculina, donde el proceso de mutilación femenino es gradual y considerado legítimo ${ }^{(9)}$. De esta forma, la hegemonía del sistema patriarcal se refleja en las relaciones entre las personas y en las relaciones entre hombres y mujeres organizados socialmente como parejas, reforzando asimetrías de poder(19).

Sin embargo, a pesar de no se confirmar explícitamente la violencia física, 11 mujeres se sentían controladas constantemente por sus parejas y $24,7 \%$ de las alumnas expresaron la ocurrencia de violencia psicológica, cuando mencionaron que sus parejas las criticaban y humillaban en público o en privado. En este sentido, las investigaciones sobre la violencia física contra mujeres en relacionamientos íntimos concluyen que esta es casi siempre acompañada de violencia psicológica y constituye entre un tercio y la mitad de los casos de violencia sexual(21)

En cuanto a la violencia sexual, solamente $1,4 \%$ de las entrevistadas manifestaron que a veces sus parejas las obligaban a realizar actos sexuales. Se verifica que la violencia sexual fue poco frecuente, pero está conjugada a otros tipos de violencia(13), especialmente la violencia psicológica.

En lo que se refiere al consumo de alcohol, más de la mitad de las participantes (57.5\%) consumían alcohol, especialmente en reuniones sociales. En cuanto a sus parejas, el $67,1 \%$ consumían alcohol también en reuniones sociales; los resultados no demuestran señales de adicción al alcohol por las participantes o sus parejas. Como es común encontrar en la literatura la afirmación de que el uso de alcohol u otro tipo de droga por parte del agresor es un desencadenador de actos de violencia(9), la no adicción al alcohol por las alumnas y sus parejas puede indicar un factor protector contra la violencia y dar base a la constatación, en esta investigación, de que los niveles de violencia física son bajos.

Considerando también los bajos índices de violencia física encontrados en esta investigación y que usualmente causan la búsqueda de ayuda externa(19), las entrevistadas no buscaron ayuda de la policía, una vez que no consideraron haber sufrido ninguna violencia grave. Sin embargo, el $41,1 \%$ de las participantes no conocía sus derechos con relación a la violencia doméstica. Este índice es preocupante pues el conocimiento de sus derechos es una condición para el ejercicio de la ciudadanía por parte de estas mujeres.

En suma, los resultados del estudio no demostraron la presencia de violencia física significativa contra las alumnas de la carrera de Enfermería, por parte de sus parejas. No obstante, hay señales de violencia psicológica, que son más sutiles y no tan visibles como la violencia física, pero que afectan la salud de las mujeres $y$, como consecuencia, deben ser consideradas por los servicios y profesionales de salud(20).

\section{Consideraciones Finales}

En la actualidad se debate la expansión de la violencia al mismo tiempo en que se afirman, en diferentes acuerdos internacionales y legislaciones nacionales, los derechos humanos civiles y sociales. La violencia es identificada en espacios públicos y privados, en las relaciones institucionales, grupales o interpersonales. La asimetría entre la consolidación de derechos y el aumento de la violencia se basa en la convicción de que la libertad de ejercicio de los derechos humanos significa centrarse en cuestiones individuales, lo que resulta en la "cosificación" del otro y causa una ruptura en las relaciones humanas que pasa a justificar las violencias.

En el ámbito de las diferentes formas de violencias, la violencia contra la mujer causada por su pareja se encuadra en el contexto de una asimetría de poder entre los géneros, muchas veces fundamentada en la idea de que las mujeres tienen menos derechos que los hombres, o que son "objetos" de los hombres. Importa resaltar que entre los factores de riesgo para la violencia doméstica contra la mujer está el consumo de alcohol. Los resultados de este estudio indicaron un bajo nivel de violencia doméstica sufrida por las alumnas de la carrera de Enfermería y Obstetricia de la Universidad de Guanajuato, México, con un alto consumo de alcohol entre las entrevistadas y sus parejas, pero no se observó la adicción.

Es importante resaltar que por ser estudiantes universitarias, se esperaba un nivel mayor de conocimiento acerca de sus derechos. Los resultados apuntan que cerca de $41 \%$ de las alumnas entrevistadas no conocían sus derechos humanos. La realidad de la dominación y violencia contra la mujer solamente va a cambiar cuándo las propias mujeres conozcan sus derechos y los medios para ejercerlos en su día a día. De esta forma, se considera necesaria la realización de acciones de concientización de las mujeres en lo que se refiere a sus derechos, así como sobre las posibilidades de buscar ayuda legal y del Estado en casos de violencia. 


\section{Agradecimientos}

Agradecemos a la Comisión Interamericana para el Control del Abuso de Drogas/CICAD de la Secretaria de Seguridad Multidimensional/SSM de la Organización de los Estados Americanos/OEA, la Secretaria Nacional de Políticas sobre Drogas/SENAD do Gabinete de Seguridad Institucional/Brasil, la Escuela de Enfermería de Ribeirao Preto de la Universidad de Sao Paulo y Centro
Colaborador de la Organización Mundial de la Salud para el Desarrollo de la Investigación en Enfermería, la población representada en los estudios de investigación, bien como a las autoridades de las universidades representadas por los participantes del Programa En-Line de Especialización en Investigación sobre el Fenómeno de las Drogas PREINVEST, periodos 2005, 2006, 2007 y 2008.

\section{Referencias}

1. Montagner MÂ, Amorim RF, Silva JG, Lira AVG. Violência e saúde. Ciênc Saúde Colet. abril 2008;13:805-6.

2. De Canales FH. Metodología de la Investigación: Manual para el desarrollo de personal de salud. México: Limusa; 2007. p. 327.

3. Valdebenito HC. María da Penha: Violencia contra a mulher no Brasil. Rev Acta Bioetica. jun 2007;13(1):133.

4. Organización Mundial de la Salud - OMS [Internet]. ¿Que es una Droga?. [acesado en: 22 febrero 2007]. Disponible en: http://members.fortunecity.es/kaildoc/queesdroga.htm

5. United Nations Office on Drugs and Crime - UNODC. 2008 World Drug Report. Austria: UNODC; 2008.

6. Roldán MCB, Galera SAF. Perception of the mothering role of women who live in a context of drugs and violence. Rev. LatinoAm. Enfermagem. dezembro 2005; 13(2):1118-26.

7. Rabello PM, Caldas AF Jr. Violência contra a mulher, coesão familiar e drogas. Rev Saúde Pública. dezembro 2007; 41(6): $970-8$

8. Schraiber LB, Oliveira AFPLDO, França-Junior I, Diniz S, Portella AP, Ludermir AB, et al. Prevalência da violência contra a mulher por parceiro íntimo em regiões do Brasil. Rev Saúde Pública. outubro 2007; 41(5):797-807.

9. Galvão EF, Andrade SM. Violência contra a mulher: análise de casos atendidos em serviço de atenção à mulher em município do Sul do Brasil. Saúde Soc. maio-ago. 2004; 13(2):89-99.

10. Schraiber LB, Oliveira AF, França I, Pinho AA. Violência contra a mulher: estudo em um unidade de atenção primária à saúde. Rev Saúde Pública. agosto 2002;36(4):470-7.

11. Klevens J. Violencia física contra la mujer en Santa Fe de Bogotá: prevalencia y factores asociados. Rev Panam Salud Pública. 2001; 9(2):78-83.

12. Grossi K. Violência contra a mulher: implicações para os profissionais de saúde. In: Loopes MJM, Meyer DE, Waldow VR. Gênero e Saúde. Porto Alegre (RS): Artes Médicas; 1996. p. 133-49.
13. Schraiber LB, Oliveira AFPL, Couto MT. Violência e saúde: estudos científicos recentes. Rev Saúde Pública. agosto 2006; 40(no esp):112-20.

14. Suárez L, Menkes C. Violencia Familiar ejercida en contra de Ios adolescentes mexicanos. Rev Saúde Pública. agosto 2006; 40(4):611-9.

15. Guerra C. Violência e poder nas relações de gênero - as cenas. Cad Espaço Feminino. jan 1997; 4(4/5):5-27.

16. Araceli QT. Violencia en la mujer como estimulo definitivo em el nivel de adaptación em el modo de autoconcepto [tesis de maestría]. Celaya (Guanajuato): Facultad de Enfermería y Obstetricia de Celaya; 2007.

17. Heise L, Pitanguy J, Germain A. Violence against women: the hidden health burden. Washington (DC): International Bank for Reconstruction and Development; 1994.

18. Organización Panamericana de la Salud. Violencia contra la mujer: un tema de salud prioritario. Washington (DC): División de Salud Familiar Reproductiva; División de Salud y Desarrollo Humano; 1998.

19. Deeke LP, Boing AF, Oliveira WF, Coelho EBS. A Dinâmica da violência doméstica: uma análise a partir dos discursos da mulher agredida e de seu parceiro. Saúde Soc. 2009; 18(2):248-58.

20. Watts C, Zimmerman C. Violence against women: global scope and magnitude. Lancet. 2002; 359(9313):1232-7.

21. Kronbauer JFD, Meneghel SN. Perfil da violência de gênero perpretada por companheiro. Rev Saúde Pública. 2005; 39(5):695-701.

22. Silva $\mathrm{J}$ da, et al. Illicit drug use in seven Latin American countries: critical perspectives of families and familiars. Rev. Latino-Am. Enfermagem. 2009; 17(nº spe):763-9.

23. Fonseca RMGS da, et al. Domestic violence against women from the perspective of the community health agent. Rev. LatinoAm. Enfermagem. 2009 Dec; 17(6):974-80.

24. Penna LHG, Carinhanha JI, Leite LC. The educative practice of professional caregivers at shelters: coping with violence lived by female adolescents. Rev. Latino-Am. Enfermagem. 2009 Dec; $17(6): 981-7$.

Recibido: 24.9.2008

Aceptado: 2.3.2010 